\title{
INSTALLATION EN CONCUBINAGE EN ESPAGNE ET EN FRANCE
}

L'objet de cet article est de montrer que les jeunes Français et Espagnols suivent des processus de construction identitaire différents. Nous partirons de l'exemple des jeunes concubins des deux pays. Les jeunes Français développent davantage leur identité personnelle que les Espagnols, lesquels ont une plus forte identité statutaire. Les premiers se construisent dans des logiques d'autonomie et d'insécurité affective et matérielle alors que les seconds le font dans des logiques de protection et de sécurité.

Sandra Gaviria

Maître de conférences à PIUT du Havre, département carrières sociales, membre du CIRTAl et membre associé du CERLIS

IUT du Havre

Département carrières sociales

Place Robert Schumann

BP 4006

76610 Le Havre

Tél. : 0132744727

Courriel : sandra.gaviria@univ-lehavre.fr 
Les jeunes Français et Espagnols suivent des processus différents de construction identitaire jusqu'au moment d'une totale autonomie et indépendance vis-à-vis de leurs parents. K. Chaland' donne cette définition, que reprend F. de Singly ${ }^{2}$ : a L'indépendance, et notamment l'indépendance économique, est la manière dont l'individu peut, gråce à ses ressources personnelles, tirées de son activité, moins dépendre de proches : l'autonomie est la maîtrise du monde dans lequel cette personne vit : monde défini par l'élaboration soit de règles personnelles, soit, en cas de vie commune, de règles construites dans la négociation à plusieurs. Lorsque ces deux dimensions sont réunies - indépendance et autonomie -, alors l'individu moderne a le sentiment d'être libre, au moins dans sa vie privée. Cette liberté est précieuse, elle condense la totalité du processus de I'individualisation, avec l'authenticité, l'indépendance et l'autonomie 2 . x Les jeunes partent de chez leurs parents non seulement à des moments différents lles Français le font plus tôt que les Espagnols) mais de manière différente. Les jeunes concubins espagnols ont une forte identité personnelle si nous les comparons aux autres jeunes Espagnols, mais si nous les comparons aux jeunes Français, on observe qu'ils ont une forte identité statutaire. À travers l'étude des concubins des deux pays, nous mettrons en relief les différents processus de construction identitaire des jeunes.

Cette recherche s'inscrit dans le cadre d'une sociologie compréhensive. Les résultats exposés ici sont issus d'un travail de recherche au cours duquel soixante entretiens semi-directifs ont été réalisés avec des jeunes de Madrid et de Paris. Le but était de comprendre le sens que les jeunes donnaient à leur action. Le profil des interviewés a été le suivant : jeunes de 25 à 30 ans, qui ont un emploi et qui résident soit chez leurs parents, soit en concubinage, soit avec leur époux ou épouse. C'est une recherche comparative dans laquelle nous avons surtout mis en avant les différences. « Voir l'Europe de près, de très près, déforme la perception d'ensemble, et rend le spectateur surtout sensible aux différences ${ }^{4}[\ldots]$. .

En Espagne, $70 \%$ des jeunes partent de chez leurs parents au moment du mariage, alors qu'en France la plupart des jeunes partent de chez leurs parents puis vivent dans des situations diverses (seuls, en concubinage, en colocation) jusqu'à ce qu'un jour ils décident de se marier. Ceci signifie que, parmi les jeunes de 25 a 30 ans qui vivent en concubinage dans les deux pays, les Espagnols représentent une exception à la norme : $8 \%$ en 1999 (INJUVE, 2000), alors que les Français sont dans la norme de leur pays : $30 \%$ en 1999 (INSEE, 2000). Nous développerons surtout le cas de l'Espagne en opérant une mise en perspective avec la France.

1. Chalano K, individualisation et transformations de la sphere privé : le discours sur l'individu et la famille, université Marc Bloch, Strasbourg, thése de doctorat de sociologie (dir. P. Watier), Strasbourg, 1999.

2. DE SINGLY F., * La naissance de l'individu individualisé et ses effets sur la vie conjugale et familiale ", in DE SinGLY F. (dir.h, Etre soi parmi los autres : famille et individualisation, L'Harmattan, coll. * Logiques sociales a, Paris, 2001, t. L, pp. 5-14.

3. Ibid., p.11.

4. Commalle J, DE Singly F. (dir.), La question familiale en Europe, L'Harmattan, coll. * Logiques sociales ^, Paris, 1998. 


\section{LES JEUNES ESPAGNOLS}

Avant l'installation en concubinage, les jeunes suivent deux types de trajectoire; soit ils partent de chez leurs parents pour vivre à deux, soit ils ont vécu peu de temps en dehors de chez leurs parents avant de s'installer en couple. Dans les deux cas de figure, ils ont passé des années à vivre avec leurs parents, se construisant auprès d'eux, en ayant une sécurité affective et matérielle. Le jour du départ, généralement, ils ont un emploi stable et des économies.

Dans le premier cas de figure, les jeunes passent de la vie de famille à la vie de couple. Ils partent pour s'installer avec la personne avec laquelle ils comptent faire leur vie.

Dans le deuxième cas de figure, les jeunes n'ont pas vécu longtemps hors de chez leurs parents (un an ou deux), ils n'ont pas connu plusieurs villes, ni des modalités de vie variées (seuls, en concubinage...). Souvent, ils ont vécu en colocation et, pour la plupart, ils n'ont jamais vécu seuls avant l'installation en couple. La vie en solo est peu appréciée par les jeunes Espagnols; ils ne sont d'ailleurs que $1 \%$ des jeunes de 25 à 29 ans, en 1996, à la choisir (INSEE, 2000). Les justifications sont diverses, mais de manière générale, il y a l'idée que celui qui vit seul est seul affectivement. Ce mode de vie n'est pas considéré comme permettant aux jeunes de développer leur identité personnelle mais apparaît comme celui choisi par les jeunes " bizarres „ou vivant dans des familles oủ règne un mauvais climat.

\section{PRENDRE LA DÉCISION}

Les jeunes réfléchissent en amont, et non pas en aval, avant de s'installer ensemble. Ils n'ont pas vécu préalablement l'un chez l'autre lorsqu'ils ont décidé de vivre en concubinage. Dans la plupart des cas, l'un des membres du couple - parfois les deux - vivait chez ses parents.

En ce qui concerne la décision de vivre ensemble, nous pouvons distinguer deux groupes de jeunes.

Dans le premier groupe, la décision a été mûrie et prise longtemps avant l'installation. L'un des signes en est l'achat préalable d'un logement en commun et le remboursement déjà effectué d'une partie de l'emprunt. C'est le cas d'Asun. Celle-ci sort avec son petit ami depuis dix ans et vit en concubinage depuis deux ans. Elle a attendu d'avoir suffisamment économisé avant de sauter le pas. Sara sort avec son petit ami depuis quatre ans, mais elle avait épargné pour l'achat d'un logement lorsqu'elle était célibataire. Ces jeunes habitaient chez leurs parents avant de vivre en couple, ils n'ont pas considéré qu'il était important de faire au préalable une expérience de vie en solo ou indépendante.

Dans le deuxième groupe, la décision survient à la suite d'un concours de circonstances. Souvent, l'un des membres du couple vivait de manière autonome au moment de prendre la décision de la vie en commun. Raquel vivait en colocation et ne s'entendait pas avec ses colocataires, elle voulait déménager. A ce momentlà, elle et son petit ami se sont dit que ce serait bien d'emménager ensemble. Son petit ami habitait chez sa mère et ne pouvait pas vraiment rester dormir chez elle:

5. Kaurmawn J.C., La femme seule et le prince charmant : enquéte sur la vie en solo, Nathan, coll. " Esaais et recherches. Sciences sociales *, Paris, 1999. 
* La mère de mon copain est une dame très âgée et la situation n'était pas facile. II dormait chez moi, mais il devait rentrer chez lui à 6 heures du matin pour faire semblant d'avoir dormi chez sa mère. La situation était intenable. " Juan vivait dans un appartement avec des amis mais tous allaient partir : $\alpha$ Je vivais avec des amis, mais chacun partait de son côté et je ne savais pas très bien quoi faire. Ma copine avait pris la décision de partir vivre seule. Alors on s'est dit: "Bon, on s'entend très bien, tout est très beau, pourquoi ne pas tenter quelque chose ?" "

Les jeunes préviennent leurs parents avant de vivre en concubinage car ils ont besoin de savoir que leurs parents * acceptent " leur décision, du moins qu'elie ne les fache pas trop. Annoncer la décision aux parents révèle un fort sentiment d'appartenance familiale. Les enfants considerent que leurs parents doivent donner leur avis, Lorsque la décision n'est pas annoncée aux parents avant l'installation, c'est que les parents ne sont pas du tout au courant de la situation : c'est le cas de Carmen dont les parents ignorent qu'elle vit en couple. Carmen ne veut pas être mal jugee par ses parents car elle sait qu'ils ne trouveraient pas cela bien et ne pourraient pas le comprendre : « Ils habitent un village et ils sont conservateurs ; là-bas je pense que deux personnes ne doivent pas vivre en concubinage, tout le monde se marie. Ce sont des mariages à trois cents invités. " De plus, ses parents ont toujours eu d'elle l'image de quelqu'un vivant hors norme, c'est pourquoi Carmen ne veut pas les conforter dans cette idée. Parfois, les parents ne sont pas complètement dupes, mais font semblant de ne rien soupçonner.

Avant l'installation en couple, les parents rencontrent le partenaire de leur enfant, comme s'ils devaient donner leur accord. Nous n'avons pas trouvé de cas où les parents ne connaissaient pas au préalable le conjoint, Un jeune qui s'installe en couple avant de présenter son conjoint à la famile est mal vu par celle-ci : c'est comme s'il la rejetait. Le couple formé par le jeune concerne aussi la famille.

\section{L'ATTITUDE DES PARENIS}

Lorsqu'ils apprennent la décision de concubinage et par la suite également, les parents et les beaux-parents donnent leur avis. Dans certains cas, des jeunes qui comptaient vivre en concubinage en abandonnent l'idée à la suite des réactions des parents et ils se marient. Lorsque les jeunes passent outre les recommandations des parents, ils subissent des pressions familiales pour qu'ils se marient au plus vite.

Nous pouvons distinguer quatre groupes de parents selon leur attitude face à la décision de concubinage.

Le premier groupe est formé par les parents qui font du chantage économique et affectif à leur enfant pour qu'il se marie sans vivre en concubinage. Pour certains jeunes, il est important de faire les choses dans le cadre d'une bonne ambiance familiale et ils sont prêts à cet effet à abandonner leurs projets.

Le deuxième groupe comprend les parents qui essaient de faire pression pour parvenir à ce que le couple se marie. A. de Miguel et M. Escuin montrent comment, en Espagne, dans les enquêtes d'opinion, les gens expriment leur acceptation du concubinage comme « valeur $n$, mais que, lorsqu'il s'agit de leur

6. DE Micuel A., Esculv M., ABC de la opinión espariola, Espasa, Madrid (Espagne), 1997. 
propre enfant, la réaction n'est pas la même. Les gens n'aiment pas ce choix de vie. Les parents de Carmelo sont contre le fait qu'il habite avec sa petite amie sans être marié. L'annonce de sa décision a été un moment très dur car il savait qu'il allait faire de la peine à ses parents : $\alpha$ J'ai eu beaucoup de mal à l'annoncer à mes parents, parce que je savais qu'ils allaient être tristes. Je savais qu'ils n'allaient pas aimer du tout parce qu'ils sont très catholiques. Déjà, dans un village, c'est difficile ; avec mes parents c'était d'autant plus dur en raison de leurs croyances. C'est pour ça que j’ai eu beaucoup de mal à leur dire. * II est curieux de constater que les parents de Carmelo ne sont pas furieux ou fåchés, mais tristes. Peut-être estce là une manière de faire du chantage affectif à leur enfant? Lorsque Carmelo et sa petite amie vont dans leur village d'origine (lieu de résidence de leur famille à tous deux), chacun dort chez soi.

Le troisième groupe est formé par les parents qui sont déçus et souhaitaient le mariage de leur enfant. Certains respectent relativement leur enfant et son couple, c'est-à-dire qu'ils ont donné leur avis au début mais ne disent plus rien par la suite. Beatriz explique que, dans un premier temps, sa mère lui a fait du chantage affectif : a Mes parents imaginaient plus ou moins que j'allais partir vivre à Madrid pour rejoindre mon copain parce qu'on avait acheté l'appartement. Ils pensaient qu'une fois les travaux de l'appartement achevés, j'allais prendre la décision de me marier et non simplement de vivre avec lui. Lorsque je leur ai annoncé que je n'allais pas me marier, mon père l'a très bien pris, ma mère, elle, a eu de la peine, elle m'a dit que je faisais une erreur. Les premiers mois, elle était un peu froide au téléphone, mais par la suite, tout s'est très bien passè. \$ D'autres font pression jusqu'à ce que les jeunes concubins se marient. C'est le cas des beauxparents de Saul : ils voulaient que leur fille Carmen, qui était contre le mariage, se marie avec son ami et l'ont obtenu. Saul raconte: " Pour les parents de Carmen, ma femme, le fait qu'on habite en concubinage avait été un peu traumatisant. IIs ne l'acceptaient pas bien. C'est moi qui ai poussé pour le mariage parce que je ne voulais pas donner de l'importance à ce sujet. Je voulais trouver la solution la moins traumatisante possible pour tout le monde. Alors je me suis dit que c'était la meilleure solution. $n$

Le dernier groupe est formé par les parents qui approuvent que leur enfant habite en concubinage ou qui, du moins, ne donnent pas un avis négatif. Juan (28 ans) prétend que ses parents auraient été surpris par un mariage précipité. II explique comment il a annoncé sa décision à ses parents : « Avant de partir, je l'ai dit à mes parents. J'ai écouté ce qu'ils me disaient et j'en ai tenu compte parce que c'était la première fois que j'allais le faire et, pour moi, c'était une nouvelle expérience, alors c'était intéressant d'entendre ce qu'ils avaient à me dire. Ils ont essayé de voir si c'était clair dans ma tête, parce que, disaient-ils, c'est une décision très importante. Et bon, comme ils ont vu qu'on savait ce qu'on faisait et qu'on n'avait pas des oiseaux dans la tête, ils ont dit: "Eh ben vas-y I"

Les différentes stratégies que les parents utilisent avec leurs enfants montrent souvent qu'ils ne respectent pas leur choix de vie et qu'ils essaient de les faire changer d'avis par tous les moyens possibles. Les jeunes, de leur côté, ont une forte identité personnelle car ils persistent dans leur choix, du moins à court terme, en dépit des pressions parentales. Néanmoins, ils acceptent la pression et les contraintes sans se fâcher ou s'y opposer. 
L'analyse de l'attitude des parents à travers le discours des jeunes montre que les parents associent sécurité à mariage et que n'importe quelle autre forme de vie est moins souhaitable parce qu'elle entraîne plus d'insécurité. Nous observons chez eux une logique forte et une grande force du groupe familial qui essaie de conserver une emprise sur ses membres et sur les trajectoires de vie. Les parents ne respectent pas la vie privée des jeunes et considèrent que leur choix de vie les concerne. M. Rico et X. Roigé? ont montré que les parents, même lorsqu'ils acceptent la cohabitation de leurs enfants, la voient comme une forme incomplète d'émancipation, comme s'il s'agissait de longues fiançailles ${ }^{8}$. Ceci pourrait expliquer la pression exercée par certains parents. Le concubinage de leur enfant est mal ressenti car ils se vivraient comme des mauvais parents : l'enfant n'est ni au domicile familial, ni marié.

\section{Les jeunes Espagnols préviennent leurs parents avant de vivre en concubinage car ils ont besoin de savoir que leurs parents " acceptent " leur décision.}

Les parents ne considerent pas leurs enfants comme des individus individualisés mais comme des membres de la famille devant agir en accord avec les attentes familiales et selon leur identité statutaire. Les jeunes subissent non seulement une pression sociale, car ils sont une exception à la norme, mais également une pression familiale. Ils pourraient choisir de se fâcher avec leurs parents en raison du manque de respect envers eux, mais ils ne le font pas. II semble que, pour eux, la préservation de l'harmonie familiale soit préférable au conflit. On pourrait dire que, contrairement aux autres jeunes Espagnols, les concubins ont une forte identité personnelle par rapport à la famille. Neanmoins, ils ne se vivent pas comme des individus à part entière dans le sens oủ ils demandent l'avis des parents et annoncent leurs projets avant de les mettre en œuvre. Parfois ils renoncent me̊me à long terme à leur projet initial.

\section{ENSEMBLE, POUR TOUJOURS}

Concernant le sens que les jeunes donnent au concubinage, la typologie suivante nous donne des éléments sur leur construction identitaire ainsi que sur l'importance accordée à la décision d'une vie commune.

7. Rico M, Rolgt $X_{n}$, Irse de casa : relatos de emancipación en dos generaciones, Congreso de la Asociación de Demografia histórica, Portugal, 2001, p. 11.

8. Nowiazgo. 


\section{Le concubinage subi}

Certains jeunes souhaitaient se marier mais pas leur partenaire, c'est le cas de Raquel et de Beatriz. Celles-ci vivent en concubinage non parce qu'elles trouvent que c'est bien mais parce que le mariage n'est pas possible dans l'immédiat.

Les petites amies d'Eduardo et de Juan se trouvent dans une cohabitation subie car elles voulaient se marier avant de commencer une vie commune. Juan dit de sa petite amie : * Pour elle, qu'on ne soit pas mariés, c'est un souci, c'est en contradiction avec ses principes et avec son education. Elle n'est pas toujours à l'aise avec la situation. Souvent, elle me présente comme un copain et non pas comme la personne avec qui elle vit. s La petite amie d'Eduardo tient elle aussi au mariage: " Pour elle, c'est très difficile parce que ses sept frères sont mariés, ses copines sont mariées, alors elle veut se marier ; je crois que c'est plus pour son entourage que pour elle. $n$ La cohabitation serait davantage $x$ subie $n$ par les filles que par les garçons. Elles seraient plus enclines que les garçons à rechercher un mariage sans cohabitation préalable.

\section{Le concubinage comme "résistance "}

Ces jeunes savent et disent qu'ils vont finir par se marier avec le partenaire, mais ils résistent à la pression familiale ou conjugale.

Les belies-familles de Sara et d'Asun exercent une forte pression pour que le mariage ait lieu. Celies-ci avouent qu'elles vont se marier mais qu'elles n'ont pas l'intention de le faire tout de suite. Néanmoins, elles ont déjà acheté un appartement avec leur partenaire. Elles n'ont pas un discours qui défend et qui met en avant l'importance de la vie en concubinage. Juan a une petite amie qui veut absolument se marier mais lui préfẻre attendre un peu. II considère qu'il faut, avant le mariage, vivre ensemble pour voir si concrètement, dans la vie quotidienne, tout se passe bien. ॥ a emménagé avec sa petite amie dans l'idée de se marier si tout se passait bien.

\section{Le concubinage à la française}

Certains jeunes attribuent à la cohabitation un sens plus $\approx$ français $\%$, dans le sens où ils la vivent comme une expérience en elle-même, qui peut évoluer, changer et s'arrêter. A. Alabart, A. Cabré et A. Domingo ${ }^{9}$ constatent que th chez les cohabitants, la possibilité d'une rupture est permanente à l'horizon. Ainsi la relation est-elle pensée et vécue "tant qu'elle dure" ^. A nos yeux, aujourd'hui, quinze ans plus tard, cette caractéristique est propre aux jeunes de ce groupe mais ne peut pas s'appliquer à ceux des autres groupes. Les jeunes appartenant à ce groupe sont partis de chez leurs parents en étant dans une situation économique plus précaire que les autres et, souvent, avant de vivre en couple, ils ont vécu en colocation. II faut préciser que les parents n'ont pas financé leur départ, contrairement aux jeunes concubins français qui, souvent, sont soutenus par leurs parents au moment du départ de la maison familiale.

Les jeunes qui voient dans la cohabitation un acte de a résistance * et ceux qui la voient comme quelque chose de a subi $n$ ont un point commun. On pourrait dire

9. Alabart A, Caeré A., Domingo A. et al, La cohabitación en Espania : un estudio en Madrid y Barcelona, Centro de investigaciones sociológicas, Madrid (Espagne), 1988, p. 84. 
qu'ils la voient comme des fiançailles. Les fiançailles reflètent bien la manière dont les jeunes Espagnols se situent face au concubinage. Avant, lorsque les jeunes se fiançaient, cela signifiait qu'ils allaient se marier ; une rupture pouvait certes arriver, mais c'était très rare. De plus, on ne se fiançait pas plusieurs fois dans șa vie. Pour une grande partie des jeunes que nous avons rencontrés, nous notons ce rapport au concubinage : ils le vivent comme des fiançailles. Le concubinage n'est pas vécu comme une expérience parmi d'autres qui pourrait s'arrêter et reprendre plus tard avec quelqu'un d'autre, ou comme quelque chose de provisoire. II est consideré comme une phase bien déterminée entre le statut d' enfant de $x$ et celui d'u épouse/époux de w. L'enquête réalisée par I. Paris ${ }^{10}$ montre comment, en 1998 , seuls $19 \%$ des jeunes disaient souhaiter vivre en concubinage sans jamais se marier, contre $38 \%$ qui voyaient dans le concubinage une étape préalable au mariage et $39 \%$ qui n'envisageaient pas le concubinage mais uniquement le mariage. Seuls $4 \%$ des jeunes disaient ne pas souhaiter vivre en couple.

\section{Le concubinage est considéré comme une phase bien déterminée entre le statut d'" enfant de " et celui d'" épouse/époux de ".}

\section{LES JEUNES FRANÇAIS}

En France, les jeunes concubins ont des trajectoires marquées par une grande mobilité. Avant la vie à deux, ils ont vécu pendant des années en dehors du domicile parental dans des villes, des logements et sous des modalités de résidence (seuls, en colocation, en couple) différents.

Pour eux, il est important, à un moment donné, de s'éloigner de la famille et de vivre sa propre vie. Alors étudiants, dans certains cas, ils auraient pu suivre leurs études dans la ville où résidaient leurs parents, mais ils ont préféré partir, Hélène a choisi Lille car l'école y était meilleure, mais aussi pour s'éloigner de ses parents avec lesquels elle avait pourtant de bons rapports : " C'était aussi un moyen d'être indépendante, parce que mes parents étaient un peu protecteurs. Ma mère n'a jamais travaillé, elle s'est toujours focalisee sur ses enfants. Mes parents ont toujours accordé beaucoup d'importance à notre réussite au sens figuré et au sens propre parce qu'ils ont dépensé beaucoup d'argent. C'est vrai que si j'ai choiși Lille, qui est assez éloignée de Bordeaux, c'est aussi, inconsciemment ou consciemment, pour acquérir mon indépendance, $\%$ Nathalie a fait une école de commerce à Lille. Lorsqu'on lui demande s'il y avait une école située dans la ville oủ elle habitait, elle répond: * It y en avait une, mais je n'avais pas envie d'y aller et j'ai loupé l'entretien d'ailleurs [rires]. J'avais envie de partir quoi, 
de partir de la ville et de chez mes parents, de quitter Bordeaux. * Lorsque les jeunes quittent leurs parents, ils sont aidés financièrement jusqu'au moment où ils obtiennent une indépendance financière par leur travail.

Le mode de vie en solo est souvent choisi par les jeunes car ils lui attribuent une importance particulière comme moyen de devenir autonome. Erwan avait une petite amie qui habitait un studio, mais il voulait expérimenter la vie en solo : « J'ai pris un appartement seul parce que, même si j'étais avec Laurence, j'avais envie, au moins pour un an, de savoir ce que c'était d'habiter tout seut. n C'était pour lui une façon de se tester : " Je voulais ètre indépendant et apprendre à me débrouiller. Donc j'ai d'abord décidé de prendre un appart seul, mais c'était à 300 mètres de chez ma copine. Pendant environ un an, nous avions chacun notre appart à une station de bus l'un de l'autre. 1

Tout au long de leur parcours, les jeunes sont confrontés à des situations matérielles et affectives nouvelles qui les placent dans des logiques d'insécurité car rien n'est figé en amont. Au cours des années, ils se construisent, se découvrent et développent leur identité personnelle. Is changent non seulement de logement mais aussi de ville. Ceci signifie qu'ils abordent chaque fois des territoires inconnus et nouveaux qu'ils doivent découvrir. Ils varient aussi leur mode de vie (seuis, en colocation, en concubinage). Ils n'ont pas un projet de vie a priori mais se laissent guider par les envies du moment et les surprises que la vie leur réserve. Tout se passe comme s'ils se laissaient emporter par les circonstances de la vie. Ce ne sont pas les circonstances qui changent ou modifient leurs projets, mais elles les guident. Économiquement, les jeunes sont souvent dépendants de leurs parents pendant quelques années, jusqu'à ce qu'ils aient un travail. Ils doivent donc economiser petit à petit par la suite pour pouvoir avoir des économies. Les jeunes choisissent généralement la location et ne sont pas propriétaires de leur logement; leur trajectoire vitale ne leur en donne pas les moyens économiques.

\section{DE CHEZ L'UN A CHEZ L'AUTRE}

L'installation en concubinage est une décision personnelle des jeunes et ne concerne pas leurs parents. Ceux-ci, parfois, l'apprennent par la suite et ne connaissaient pas forcément le conjoint préalablement. Sara relate : " Ma mère ne le connaissait pas. En fait, on a commencé en janvier, il est venu habiter chez moi en juillet à la fin de son année scolaire et il est venu en vacances chez ma mère en août.

La vie commune commence à des moments différents de la relation selon les cas : elle peut commencer au bout de quatre mois comme au bout de trois ans. II semble qu'il n'y ait pas de règle. Différents paramètres entrent en ligne de compte, comme l'activité de chacun, les rapports avec les parents, le lieu de résidence ou encore la situation personnelle. Un concours de circonstances amène à la vie commune.

La décision de concubinage vient souvent confirmer une situation de fait. Les jeunes habitent depuis un certain temps en alternance entre les appartements de I'un et de l'autre jusqu'au moment d'en prendre un ensemble ou d'en laisser un. Erwan voulait expérimenter la vie en solo et il l'a fait pendant un an, mais la fré quence avec laquelle il voyait sa petite amie, qui habitait seule elle aussi, l'a amené 
à quitter son appartement pour des raisons pratiques : "Une fois sur deux je dormais chez Laurence ou elle venait chez moi, donc c'était plutôt chiant qu'autre chose parce que j'avais mes cours à préparer donc je venais avec plein de papiers chez elle et puis parfois, à 11 heures du soir, je courais chercher autre chose que j'avais oubliè. Heureusement qu'on était tout près, sinon ça aurait été invivable. x Franck explique ainsi son parcours : a Sophie a un peu insisté aussi, elle m'a un peu forcé la main, et, au fur et à mesure, je me suis un petit peu incrusté chez elle, n Comme l'explique J.-C. Kaufmann" : "Les jeunes couples se forment autour du sentiment amoureux et des relations interpersonnelles avant de penser à la fondation et à l'installation du ménage. „ Pour certains jeunes, la décision de vivre ensemble vient en aval, les jeunes commencent à aller l'un chez l'autre et, à un moment, la cohabitation devient évidente, "Les jeunes qui commencent à habiter ensemble aujourd'hui n'ont guère l'idée de fonder un foyer, ils le construisent avant que l'idée ne leur vienne ${ }^{12}, n$

\section{Les parents français valorisent le fait que leurs enfants fassent leur propre expérience de vie avant de s'installer en couple et qu'ils habitent en concubinage avant de se marier.}

Les parents français valorisent le fait que leurs enfants fassent leur propre expérience de vie avant de s'installer en couple et qu'ils habitent en concubinage avant de se marier. Comme le montre F. de Singly ${ }^{13}$, aux yeux des parents, le concubinage est une manière pour le jeune d'avoir sa vie personnelle tout en suivant sa trajectoire scolaire correctement. La meilleure éducation serait celle qui favoriserait pour les jeunes le test d'une palette de situations, Les parents voient d'un bon ceil que les jeunes se construisent progressivement au lieu de quitter le nid familial une fois tout $*$ acquis $n$, materiellement, économiquement et affectivement. Souvent les parents financent le départ de leurs enfants ${ }^{10}$.

11. Kaufmann J-C., La trame conjugale ; analyse du couple par son linge, Nathan, coll, "Essais et recherches. Sciences sociales ", Paris, 1992.

12. Ibid.

13. DE Singly $F_{*}$ * L'union libre : un compromis s, Dialogue, $n^{*} 92,2 *$ trimestre 1986, pp. 54-65.

14. GALLAND O., * Adolescence, postadolescence, jeunegse : retour sur quelques interprétations *, Revue française de sociologie, $n^{\circ} 4$, vol. 42, 2001, pp. 611-640. 


\section{CONCUBINS, TANT QUE GA DURE}

Lorsque les jeunes s'instalient en concubinage, ils ne savent pas si c'est avec ce conjoint qu'un jour ils se marieront ou feront des enfants. La manière dont la décision de concubinage est prise montre que la relation peut changer, s'arre̊ter et reprendre, avec la mëme personne ou quelqu'un d'autre. Comme si la vie commune elle-même déterminait la suite. Le cas de Raphaël et sa petite amie est significatif. Ils habitaient ensemble à Lille puis se sont séparés, lui est parti à Clermont et elle à Rome pour ensuite se retrouver à Paris. S. Chalvon-Demersay's montre comment, parfois, lorsqu'il y a une rupture de la vie commune, les jeunes rentrent chez leurs parents. Elle explique que tout se passe "comme si l'émancipation des enfants n'était pas absolument irréversible et que la parenté continuait, un certain temps, à proposer des filets de secours ${ }^{16} x$. C'est cette notion de réversibilité qui caractérise la cohabitation des jeunes couples vingt ans après. Il est bon que les jeunes découvrent et développent leur identité personnelle par la pratique

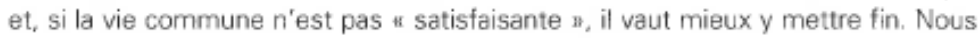
constatons encore aujourd'hui que " le couple est intense mais précaire ${ }^{17}$. Ceci fait partie de la construction identitaire des jeunes; l'important est d'étre soime̊me, de se découvrir et d'avancer selon le vécu de chacun mais non pas de tracer des projets en amont.

Pour une minorité de jeunes, le concubinage est considéré comme une étape préalable au mariage. M. Bozon ${ }^{18}$ distingue deux cas de figure concernant les formes d'entrée dans la vie conjugale, le * mariage direct " et le * mariage après la cohabitation $\mathrm{n}$. On pourrait penser à une autre formule qui serait le a mariage

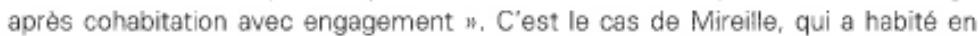
concubinage mais avec un engagement de mariage. Pour elle, le concubinage en soi n'a pas d'intérêt : "Je n'avais pas envie de m'installer dans une situation hybride, parce que pour moi, c'était hybride de vivre avec quelqu'un sans m'engager sérieusement avec. "s

Les jeunes adultes espagnols et français suivent des parcours différents jusqu'au moment de l'installation en concubinage. Les premiers se construisent dans des logiques de sécurité affective et matérielle et essaient de concilier leur identité personnelle avec l'identité familiale, les seconds le font dans des logiques d'insécurité affective et matérielle et privilégient le développement de leur identité personnelle.

Les jeunes Espagnols partent de la maison familiale en étant indépendants économiquement et avec des économies. Avant de vivre en concubinage, ils n'ont jamais vécu seuls et parfois même jamais en dehors de la maison des parents.

15. Chalvon-Demersay $\mathrm{S}_{4}$ " Le temps des ruptures : désunions libres $x_{4}$ Dialogue, $n^{\circ} 92,2^{*}$ trimestre 1986, pp. 105-115.

16. Ibid., p. 113.

17. Ibid., p. 113.

18. Bozon M., * Le mariage ; montée et déclin d'une institution n, in DE SINGLY F. (dir.), La famille, l'état des savoirs, La Découverte, coll. " Textes a l'appui. Sociologie ", Paris, 1991, p. 63. 
Lorsqu'ils s'en vont, ils n'ont pas vécu des modalités de vie différentes (seuls, en colocation, en concubinage...). Ils se sont construits pendant des années dans la proximite familiale. Au moment de la décision de vie en concubinage, ils attribuent majoritairement à la vie commune un sens pour toujours. Ils considèrent que l'avis de leurs parents est nécessaire et même important. Lorsque la réaction est trop brusque, ils se marient ou cèdent au mariage après quelques mois à peine en concubinage. Rares sont ceux qui passent outre l'avis de leurs parents sur le long terme. Les autres conservent une forte identité statutaire.

En France, les jeunes passent des années à se construire librement et développent leur identité personnelle. Ils vivent en concubinage ou avec des amis dans des villes différentes et sans économies. Le jour où ils s'installent en couple, ils ne sont pas forcément indépendants économiquement de leurs parents, mais ils ne leur demandent pas leur avis. La vie du jeune ne concerne que lui-me̊me et non pas ses parents. Le concubinage est vécu comme une expérience en soi dont le vécu déterminera la suite. 



